\title{
Multi-Drug Resistant Gram-Negative Infections Among Critically III Patients: Analysis of Baseline Characteristics and Factors Associated with Mortality
}

Fadi Aljamaan ( $\square$ faljamaan@ksu.edu.sa )

King Saud University and King Saud University Medical City https://orcid.org/0000-0001-8404-6652

Esraa Altawil

King Saud University Medical City

Mohamad-Hani Temsah

King Saud University

Ahmad Almeman

Qassim University

Research

Keywords: multidrug-resistant Gram-negative, CRE, Pseudomonas, Acinetobacter, mortality, critical illness

Posted Date: December 17th, 2020

DOl: https://doi.org/10.21203/rs.3.rs-129355/v1

License: (c) (1) This work is licensed under a Creative Commons Attribution 4.0 International License.

Read Full License 


\section{Abstract}

\section{Background}

Bacterial infections are a frequent cause of hospitalization and a leading cause of death, particularly with the emergence of antibiotics resistance. The emergence of Carbapenem resistance among gram-negative bacteria (GNB) is one of the evolving alerts as its use is considered the last resort of treatment [1].

Therefore, this urged studying the risk factors for the development of multi-drug resistant [2] GNB, identify the clinical outcomes and factors associated with mortality, especially among critically ill patients who are expected to have the worst outcomes.

Materials/methods

This is a retrospective observational study of critically ill patients who had an infection with Carbapenemresistant Enterobacteriaceae (CRE), or MDR Pseudomonas aeruginosa, or MDR Acinetobacter spp. between May 2016- Nov 2018. Baseline demographics, co-morbidities, and clinical outcomes were collected and were evaluated for association with 28 days mortality.

Results

A total of 255 patients with MDR Gram-negative cultures were screened, 77 patients met the inclusion criteria. Pseudomonas aeruginosa was the most common index organism (53\% of patients), followed by Acinetobacter spp. and CRE, respectively. The mortality rate at 28 days was (59.7\%). Non-survivors were significantly older (mean age 64 vs. 44 years, $P=0.0001$ ), had significantly worse disease severity scores on ICU admission, higher incidence of chronic kidney disease (CKD) (43\% vs. $16 \%, P=0.010)$, required more continuous renal replacement therapy (CRRT) (54\% vs. $13 \% \mathrm{P}=0.0001)$, had longer hospital length of stay prior to infection (median 34 vs. 13 days, $P=0.018$ ), and required longer inotropic and vasopressors support (median 19 vs. 8 days, $P=0.0001$ ). In multivariate logistic regression the following factors were significantly associated with mortality; requirement of inotropic support [OR 10.01 (95\% Cl 1.55-64.77); $P=0.015$ ], age [OR 1.05 (95\% Cl 1.0-1.1); $P=0.01$ ], APACHE IV score on ICU admission [OR $1.03(95 \% \mathrm{Cl} 1.0-1.06) ; \mathrm{P}=0.04$ ], and ICU length of stay [OR $1.03(95 \% \mathrm{Cl} 1.0-1.06) ; \mathrm{P}=0.035$ ].

Conclusion

MDR Gram-negative infection is associated with significant in-hospital mortality among critically ill patients. Old age, high APACHE IV score, higher ICU length of stay, and higher hemodynamic support are associated with higher mortality.

Trial registration

retrospectively registered.

\section{Background}


Bacterial infections are a frequent cause of hospitalization and a leading cause of death, particularly with the devastatingly increasing antimicrobial resistance panel [3]. The lack of an antimicrobial stewardship program in many health institutions has contributed to the emergence of drug resistance partly through the malpractice of antimicrobial usage [4].

Antimicrobial resistance expanded through the different kinds of bacteria, primarily Gram-negative Enterobacteriaceae family, particularly Pseudomonas and Acinetobacter species. Gram-negative bacteria (GNB) has become more worrisome due to the emergence of strains resistant to several or even all the available antibiotics, with the lack of upcoming promising antibiotics matching the rate of resistance growth [5]. Multi-drug resistant (MDR) organisms are mainly prevalent in the healthcare settings, including outpatient facilities and general wards, but the highest prevalence, cost, morbidity, and mortality is in critical care units. It has contributed significantly to nosocomial infections leading to high financial and health care burden $[3,5]$.

The emergence of Carbapenem-resistant organisms is one of the evolving alerts as the use of carbapenems have been considered the last resort to treat such organisms for the last two decades [6]. Previous research has shown that infection with such organisms in the critical care settings is associated with high mortality, longer ICU and hospital length of stay and worse other morbidity indicators as the duration of mechanical ventilation and organ failure [5-7].

Several studies have evaluated risk factors for mortality in patients with MDR Gram-negative infections. A meta-analysis of patients with MDR Gram-negative infections found that mechanical ventilation, ICU length of stay, presence of septic shock, delay in initiating appropriate antimicrobial treatment, high disease severity scores as APACHE II, and advanced age were associated with higher mortality [5]. Specific data is still deficient and conflicting in critically ill patients regarding associated factors with mortality $[5,8-9[$.

Therefore, this urged studying the risk factors for the development of MDR GNB, identify the clinical outcomes and factors associated with mortality, especially among critically ill patients who are expected to have the worst outcomes [2, 8-10].

\section{Materials/methods}

\section{Study Population}

This is a retrospective observational study of critically ill patients who were admitted to surgical/ or medical intensive care unit in a tertiary care academic hospital between May 2015- Nov 2017 who had an infection with Carbapenem-resistant Enterobacteriaceae (CRE), or Multidrug-resistant (MDR)

Pseudomonas aeruginosa, or MDR Acinetobacter Baumani spp. Only patients determined to have active infection were included in the study, and all culture sources were incorporated to determine the presence of the bacteria of interest. Baseline demographics, co-morbidities, and clinical outcomes were collected and were evaluated for association with 28-days mortality. Approval of the research was obtained from 
the King Saud University research center Institutional Review Board (IRB) before the commencement of the study.

\section{Variables collected}

Demographic variables (including age, gender), co-morbidities, hospital admission during last 90 days, microbiologic data (including organism type, antibiotics resistance panel and source of infection), use of inotropes and vasopressors, use of steroids, mechanical ventilation and its duration, need for Continuous Renal Replacement Therapy (CRRT), disease severity scores as Acute Physiology and Chronic Health Evaluation (APACHE II and IV), hospital and ICU length of stay, and ICU and hospital mortality.

\section{Definitions}

Index culture: The first culture during ICU admission that grew CRE, MDR Pseudomonas aeruginosa, or MDR Acinetobacter Baumani spp and associated with active infection.

Index organism: The organism that was grown on the index culture.

CRE: Enterobacteriaceae that are nonsusceptible (i.e., intermediate or resistant) to a carbapenem.

MDR P. aeruginosa: Strains with intermediate susceptibility or resistance to $\geq 1$ drug in 3 of 5 of the following classes: extended spectrum cephalosporins (cefepime, ceftazidime), fluoroquinolones (ciprofloxacin, levofloxacin), aminoglycosides, carbapenems, and piperacillin or piperacillin/tazobactam.

MDR Acinetobacter spp.: Strains with intermediate susceptibility or resistance to $\geq 1$ drug in 3 of 6 of the following classes: extended spectrum cephalosporins (cefepime, ceftazidime), fluoroquinolones (ciprofloxacin, levofloxacin), aminoglycosides, carbapenems, piperacillin or piperacillin/ tazobactam, and ampicillin/sulbactam.

These definitions are adapted from the joint initiative by the CDC and the European Center for Disease Prevention and Control (ECDC) [3].

Empiric antibiotic therapy was defined as the antibiotic therapy that the patient was receiving by the end of post-index culture day 1 . Appropriate empirical antibiotics choice was defined as empiric therapy that included an agent to which the index organism was susceptible. Definitive therapy was defined as antibiotics that the patient received after the index culture results were available.

An expert review panel consisting of one clinical pharmacy specialists in critical care, one infectious disease physician, and one critical care physician, determined the presence of active infection versus colonization by reviewing the electronic medical record [2] and CDC definitions in that regard. The expert review panel assessed the adequacy of empiric therapy according to documentation in the [2].

\section{Statistical Analysis}


Descriptive analysis was used for all data. Normally distributed data were analyzed using Mean \pm Standard deviation (SD) and t-student test for differences. Whereas, in non-normally distributed data, the median was used for descriptive data and Mann-Whitney for any significant difference. Chi ${ }^{2}$ was conducted for the association when required. The significance level was set at 0.05 with 2SD and $95 \%$ confidence intervals [7].

\section{Results}

\section{Patient Characteristics and Culture Results}

Two hundred fifty-five cases were screened for inclusion criteria; 77 cases fit the criteria corresponding to an incidence of $2 \%$ of all critical care areas admissions during the study period (Figure 1 ). Study participants were predominantly male (60\%) with a mean age of 55 years. Clinical characteristics and comorbidities of included patients are summarized in Table 1.

The median hospital length of stay was 115 days, and median ICU length of stay was 66 days, $93 \%$ of the patient's required mechanical ventilation, pneumonia was the most common infection (51\%), followed by bloodstream infections (26\%) as shown in Table 2.

Pseudomonas aeruginosa was the most common index organism (53\%), followed by Acinetobacter baumani spp. (32\%) and CRE (14\%), respectively, as shown in Table 3.

\section{8-days hospital Mortality}

Twenty-eight days hospital mortality was (60\%). Non-survivors were significantly older (mean age 64 vs. 44 years, $P=0.0001$ ), had significantly higher disease severity scores on ICU admission, higher incidence of baseline chronic kidney disease (CKD) ( $43 \%$ vs. $16 \%, P=0.010)$, had longer hospital length of stay prior to infection (median 34 vs. 13 days, $P=0.018$ ), required more frequently inotropic support and longer vasopressors support, and required continuous renal replacement therapy (CRRT) more frequently (54\% vs. $13 \% \mathrm{P}=0.0001)$, (Table 3 ).

Survivors and non-survivors had no significant difference regarding the hospital, ICU length of stay, duration of mechanical ventilation, and the use of steroids at any time during admission, as summarized in Table 4.

In multivariate logistic regression for independent variables associated with 28 days mortality, the following factors contributed significantly to mortality; use of inotropes [OR 10.01 (95\% Cl 1.55-64.77); $\mathrm{P}=$ 0.015], age [OR 1.07 (95\% Cl 1.0-1.16); P=0.039], and APACHE IV score on ICU admission [OR $1.03(95 \% \mathrm{Cl}$ 1.0- 1.06); $P=0.04$ ].

Non survivors had significantly longer ICU length of stay [OR $1.03(95 \% \mathrm{Cl} 1.00,1.06) ; \mathrm{P}=0.035$ ], while they had shorter hospital length of stay [OR 0.97 (95\% Cl 0.95, 0.99); $P=0.009$ ] (Table 5). 


\section{Discussion}

This study includes one of the largest cohorts of surgical and medical critically ill patients infected with MDR GNB in comparison to previous studies [1, 10-11], and represents mostly a sick group of critically ill patients based on the disease severity scores and predicted mortality as shown in Table 1.

The studied MDR organisms' colonization prevalence rate in our study was $2.9 \%$, and the infection prevalence rate was $2 \%$ among the studied ICU population, while the incidence of infection among colonized patients was $70 \%$, highlighting the high virulence of such organism in critically ill population, previous studies showed the variable range of colonization and infection rates among colonized population highlighting the effect of population variabilities, intensivist antimicrobial usage practice and antibiotic stewardship application affecting the prevalence of MDR organisms across different critical care units [12-13].

In this study, the 28-days mortality was $60 \%$ and occurred mostly within the ICU admission; previous studies have reported variable mortality rates with MDR GNB in critical care settings ranging between 15$40 \%[5,14-17]$. Hospital-acquired pneumonia was the most common infection (51\%), even though $93 \%$ of our population required mechanical ventilation with long duration (mean= 54 days), this high rate of mechanical ventilation requirement points to the high morbidity and organ failure associated with those organisms like high requirement for renal replacement therapy and high incidence of cardiocirculatory failure evidenced by the high vasopressors/inotropic requirements (Table 2). Our findings regarding the high mortality and morbidity associated with those organisms highlight the importance of studying such organisms and their associated infection in critically ill populations more extensively due to the cost impact they exert on the healthcare system.

Non-survivors had a higher incidence of baseline CKD and were significantly older and sicker (high morbidity scores) on admission to ICU. The last two factors have been a consistent finding in many previous studies [2,18-19], which can be utilized in the future as a predictor of outcome for patients infected with such organisms on admission to ICU and to decide level care according to them or plan further additional advanced supportive care accordingly, non-survivors had a significantly longer hospital stay before ICU admission which is a variable that was not investigated thoroughly in previous studies that might play a part in dictating the outcome of those patients, this can be explained by either longer duration of colonization with those organisms giving a chance to invade the body barriers and cause infection or those patients were sicker and had depleted physiological reserves, therefore, had a more extended hospital stay before acquiring infection and deterioration till ICU admission.

Both survivors and non-survivors did not have significant difference regarding definite antibiotic therapy duration. Additionally the duration of empirical antibiotic therapy prior to definite therapy (sensitivity based therapy) was significantly shorter in non-survivors, which signifies that those patients even had targeted therapy earlier than survivors, that variable was rarely studied in literature even though it's an essential factor potentially affecting outcome in relation to pharmacological source control, therefore being a non-contributing factor in mortality in our study hints to potentially the virulence of such 
organisms, the need for a high index of suspicion of infection in colonized patient and starting empirical therapy as early as possible before systemic microbial invasiveness and florid disease manifestation appear. Both groups did not differ regarding the appropriateness of empirical antibiotic choice or the number of empirical antibiotics used for the final sensitivity of the culprit organism compared to previous studies [20]. However, an important observation in our study is that rate of the appropriate use of empiric antibiotic therapy was high in both groups reaching at least $75 \%$, which is one of the major survival driving factors. However, mortality was still high, corresponding to other factors contributed to survival like age and high morbidity score at baseline on ICU admission $[4,15,21]$.

Despite the fact that a large percentage of our cohort (43\%) received systemic steroids during admission, there was no significant difference between both groups in terms of mortality even compared to previous studies indicating that it was not a contributing factor for mortality.

After controlling for other factors associated with mortality, high APACHE IV score on admission to ICU, need for inotropic support, longer ICU length of stay, and age were the only significant factors contributing to mortality.

Source control is an important factor in clinical success in treating bacterial infections, particularly in intra-abdominal infections commonly encountered in the SICU. Our population is a mix of surgical and medical patients with a small number of surgical patients, and we had limited access to surgical source control data; therefore we could not comment on its contribution to mortality as other studies that showed this factor to decrease mortality [13].

\section{Study limitations:}

This study has several limitations. It is observational; thus, a causal relationship between variables and mortality cannot be established. It may be underpowered to detect other important differences between survivors and non-survivors as we explored many variables, and even though our sample size was not small, but it was not large enough to study many variables regarding association with mortality. Our study was a single-center, which limits its generalization. We could not give a conclusion regarding surgical source control about the outcome as our surgical population is limited. Furthermore, exploring these findings post the COVID-19 pandemic is warranted.

\section{Conclusions}

This study demonstrates that MDR Gram-negative infection is associated with significant in-hospital mortality among critically ill patients. Old age, high APACHE IV score, and higher hemodynamic support long ICU length of stay are associated with higher mortality. Further work in larger sample size is needed to distinguish between simple associations versus cause-effect relationships, but this study provides support for using these factors in further clinical research projects.

\section{Declarations}


Ethics approval and consent to participate: The study was approved by the Institutional Review Board at the College of Medicine and King Saud University Medical City (approval \# E17-2780).

Consent for publication: All authors gave their consent for publication.

Availability of data and materials: All the data for this study will be made available upon reasonable request to the corresponding author.

Competing interests: The authors declare that they have no competing of Interests.

\section{Funding: None}

Competing interests: The authors declare that they have no competing of Interests.

Authors' contributions:FA, Project conceptualization, data collection, and analysis, manuscript drafting and finalization. EA, data collection, and analysis, manuscript drafting and finalization. MHT, manuscript drafting and finalization. AAA, manuscript drafting and finalization. All authors read and approved the final manuscript.

Acknowledgment: This research has been financially supported by Prince Abdullah Ben Khalid Celiac Disease Research Chair, under the Vice Deanship of Research Chairs, King Saud University, Riyadh, Saudi Arabia.

\section{Authors' information:}

\section{Authors emails and ORCID:}

Fadi Aljamaan faljamaan@ksu.edu.sa, https://orcid.org/0000-0001-8404-6652

Esraa Altawil etaweel@live.com

Mohamad-Hani Temsah mtemsah@ksu.edu.sa, https://orcid.org/0000-0002-4389-9322

Ahmad Almeman ahmadalmeman@gmail.com

\section{References}

1. Michalopoulos A, Falagas ME, Karatza DC, Alexandropoulou P, Papadakis E, Gregorakos L, et al. Epidemiologic, clinical characteristics, and risk factors for adverse outcome in multiresistant gramnegative primary bacteremia of critically ill patients. Am J Infect Control. 2011;39(5):396-400

2. Babich T, Naucler P, Valik JK, Giske CG, Benito N, Cardona R, et al. Risk factors for mortality among patients with Pseudomonas aeruginosa bacteraemia: a retrospective multicentre study. Int $\mathrm{J}$ Antimicrob Agents. 2020;55(2):105847 
3. Maragakis LL, Perencevich EN, Cosgrove SE. Clinical and economic burden of antimicrobial resistance. Expert Rev Anti Infect Ther. 2008;6(5):751-63

4. MacVane SH. Antimicrobial Resistance in the Intensive Care Unit: A Focus on Gram-Negative Bacterial Infections. J Intensive Care Med. 2017;32(1):25-37

5. Vardakas KZ, Rafailidis PI, Konstantelias AA, Falagas ME. Predictors of mortality in patients with infections due to multi-drug resistant Gram negative bacteria: the study, the patient, the bug or the drug? J Infect. 2013;66(5):401-14

6. Lemos EV, de la Hoz FP, Einarson TR, McGhan WF, Quevedo E, Castaneda C, et al. Carbapenem resistance and mortality in patients with Acinetobacter baumannii infection: systematic review and meta-analysis. Clin Microbiol Infect. 2014;20(5):416-23

7. Ozsurekci Y, Aykac K, Cengiz AB, Basaranoglu ST, Sancak B, Karahan S, et al. Bloodstream infections in children caused by carbapenem-resistant versus carbapenem-susceptible gram-negative microorganisms: Risk factors and outcome. Diagn Microbiol Infect Dis. 2017;87(4):359-64

8. Wang W, Jiang T, Zhang W, Li C, Chen J, Xiang D, et al. Predictors of mortality in bloodstream infections caused by multidrug-resistant gram-negative bacteria: 4 years of collection. Am J Infect Control. 2017;45(1):59-64

9. Paramythiotou E, Routsi C. Association between infections caused by multidrug-resistant gramnegative bacteria and mortality in critically ill patients. World J Crit Care Med. 2016;5(2):111-20

10. Lin CC, Liu TC, Kuo CF, Liu CP, Lee CM. Aerosolized colistin for the treatment of multidrug-resistant Acinetobacter baumannii pneumonia: experience in a tertiary care hospital in northern Taiwan. $J$ Microbiol Immunol Infect. 2010;43(4):323-31

11. Katsaragakis S, Markogiannakis H, Samara E, Pachylaki N, Theodoraki EM, Xanthaki A, et al. Predictors of mortality of Acinetobacter baumannii infections: A 2-year prospective study in a Greek surgical intensive care unit. Am J Infect Control. 2010;38(8):631-5

12. Agarwal S, Kakati B, Khanduri S, Gupta S. Emergence of Carbapenem Resistant Non-Fermenting Gram-Negative Bacilli Isolated in an ICU of a Tertiary Care Hospital. J Clin Diagn Res. 2017;11(1):Dc04-dc7

13. Jarrell AS, Kruer RM, Berescu LD, Pronovost PJ, Trivedi JB. Factors associated with in-hospital mortality among critically ill surgical patients with multidrug-resistant Gram-negative infections. J Crit Care. 2018;43:321-6

14. Falagas ME, Tansarli GS, Karageorgopoulos DE, Vardakas KZ. Deaths attributable to carbapenemresistant Enterobacteriaceae infections. Emerg Infect Dis. 2014;20(7):1170-5

15. Siwakoti S, Subedi A, Sharma A, Baral R, Bhattarai NR, Khanal B. Incidence and outcomes of multidrug-resistant gram-negative bacteria infections in intensive care unit from Nepal- a prospective cohort study. Antimicrob Resist Infect Control. 2018;7:114

16. Magira EE, Islam S, Niederman MS. Multi-drug resistant organism infections in a medical ICU: Association to clinical features and impact upon outcome. Med Intensiva. 2018;42(4):225-34 
17. Parker CM, Kutsogiannis J, Muscedere J, Cook D, Dodek P, Day AG, et al. Ventilator-associated pneumonia caused by multidrug-resistant organisms or Pseudomonas aeruginosa: prevalence, incidence, risk factors, and outcomes. J Crit Care. 2008;23(1):18-26

18. Ciginskiene A, Dambrauskiene A, Rello J, Adukauskiene D. Ventilator-Associated Pneumonia due to Drug-Resistant Acinetobacter baumannii: Risk Factors and Mortality Relation with Resistance Profiles, and Independent Predictors of In-Hospital Mortality. Medicina (Kaunas). 2019;55(2).

19. Lopez-Montesinos I, Dominguez-Guasch A, Gomez-Zorrilla S, Duran-Jorda X, Siverio-Pares A, ArenasMiras MM, et al. Clinical and economic burden of community-onset multidrug-resistant infections requiring hospitalization. J Infect. 2020;80(3):271-8

20. Metan G, Sariguzel F, Sumerkan B. Factors influencing survival in patients with multi-drug-resistant Acinetobacter bacteraemia. Eur J Intern Med. 2009;20(5):540-4

21. Iregui M, Ward S, Sherman G, Fraser VJ, Kollef MH. Clinical importance of delays in the initiation of appropriate antibiotic treatment for ventilator-associated pneumonia. Chest. 2002;122(1):262-8

\section{Tables}

Table 1. Demographics, co-morbidities, and other patient characteristics. 


\begin{tabular}{|c|c|}
\hline & Overall $(\mathrm{N}=77)$ \\
\hline Age, years, mean (SD) & $55.83 \pm 19$ \\
\hline Male, n (\%) & $46(60)$ \\
\hline Female, n (\%) & $31(40)$ \\
\hline Hospital admission in the past 90 days, $\mathrm{n}(\%)$ & $16(21)$ \\
\hline Bed ridden, $\mathrm{n}(\%)$ & $18(23)$ \\
\hline Dementia, n (\%) & $19(25)$ \\
\hline Diabetes, n (\%) & $38(50)$ \\
\hline Chronic kidney disease, n (\%) & $25(32)$ \\
\hline Malignancy, n (\%) & $11(14)$ \\
\hline Hypertension, n (\%) & $38(49)$ \\
\hline Ischemic heart disease, $\mathrm{n}(\%)$ & $22(29)$ \\
\hline Cirrhosis, n (\%) & $9(12)$ \\
\hline Charlson co-morbidity score, mean \pm SD & $4.9 \pm 3.9$ \\
\hline $\mathrm{APACHE}^{*} \| \mathrm{score} 1^{\text {st }} 24$ hours in ICU, mean \pm SD & $21.8 \pm 8$ \\
\hline APACHE IV score $1^{\text {st }} 24$ hours in ICU, mean \pm SD & $86.17 \pm 26$ \\
\hline SOFA ${ }^{* *}$ score $1^{\text {st }} 24$ hours in ICU, mean \pm SD & $10.2 \pm 3.6$ \\
\hline $\mathrm{GCS}^{\star \star \star}$ on ICU admission, mean $\pm S D$ & $7 \pm 4$ \\
\hline
\end{tabular}

* Acute Physiology and Chronic Health Evaluation

** The Sequential Organ Failure Assessment

***Glasgow Coma Scale

Table 2. Patient's clinical outcomes. 


\begin{tabular}{|ll|}
\hline Variable & Outcome \\
\hline Hospital length of stay, days, median (IQR) & $68(36,131)$ \\
\hline ICU length of stay, days, median (IQR) & $39(19,81)$ \\
\hline Hospital LOS prior to index culture, days, median (IQR) & $25(10,51)$ \\
\hline 28-days hospital mortality, $\mathrm{n}(\%)$ & $46(60)$ \\
\hline Post ICU discharge mortality, $\mathrm{n}(\%)$ & $2(2.6)$ \\
\hline Hospital-acquired pneumonia, $\mathrm{n}(\%)$ & $52(51)$ \\
\hline CAUTI ${ }^{\square}, \mathrm{n}(\%)$ & $11(10)$ \\
\hline CLABSI ${ }^{\star}, \mathrm{n}(\%)$ & $27(26)$ \\
\hline Intraabdominal infection, $\mathrm{n}(\%)$ & $7(6)$ \\
\hline Soft tissue infection, $\mathrm{n}(\%)$ & $8(7)$ \\
\hline Mechanical ventilation, $\mathrm{n}(\%)$ & $72(93)$ \\
\hline Total duration of mechanical ventilation, days, mean $\pm S D$ & $53.87 \pm 74$ \\
\hline Duration of vasopressors support, days, mean \pm SD & $21.37 \pm 29$ \\
\hline Use of inotropes, $\mathrm{n}(\%)$ & $29(38)$ \\
\hline Use of steroids anytime during admission, $\mathrm{n}(\%)$ & $33(43)$ \\
\hline CRRT ${ }^{\star *}, \mathrm{n}(\%)$ & $29(38)$ \\
\hline
\end{tabular}

\# Catheter associated urinary tract infection

*Central line-associated bloodstream infection

** Continuous renal replacement therapy

Table 3. Index culture information. 


\begin{tabular}{|ll|}
\hline Variable & Overall (N=77) \\
\hline Index MDR organisms, $\mathrm{n}(\%)$ & $11(14)$ \\
CRE & $41(53)$ \\
Pseudomonas aeruginosa & $25(32)$ \\
Acinetobacter spp. & \\
\hline Specific index CRE organism, $\mathrm{n}(\%)$ & $9(82)$ \\
Klebsiella pneumonia & $1(9)$ \\
Proteus mirabilis & $1(9)$ \\
Serratia marcescens & \\
Index culture source, $\mathrm{n}(\%)$ & $52(51)$ \\
Respiratory & $27(26)$ \\
Blood & $11(10)$ \\
Urine & $8(7)$ \\
Wound & $7(6)$ \\
Intra-abdominal & \\
\hline
\end{tabular}

Table 4: Patient characteristics, survivors versus Non-survivors. 


\begin{tabular}{|c|c|c|c|}
\hline Variable & $\begin{array}{l}\text { Survivors } \\
(n=33)\end{array}$ & $\begin{array}{l}\text { Non- } \\
\text { survivors } \\
(n=44)\end{array}$ & P-value \\
\hline Age (years), mean & 44 & 64 & 0.0001 \\
\hline Charlson comorbidity score, mean & 2.3 & 6.6 & 0.0001 \\
\hline APACHE II score $1^{\text {st }} 24$ hours in ICU, mean & 18 & 24 & 0.0001 \\
\hline APACHE IV score $1^{\text {st }} 24$ hours in ICU, mean & 72 & 95 & 0.0001 \\
\hline SOFA score within $1^{\text {st }} 24$ hours in ICU, mean & 9 & 11 & 0.005 \\
\hline Hospital admission in the past 90 days, $n$ (\%) & $5(16)$ & $11(24)$ & 0.415 \\
\hline GCS on ICU admission, mean & 7 & 6 & 0.300 \\
\hline Presence of infection on ICU admission, n (\%) & $20(64.5)$ & $32(69.5)$ & 0.544 \\
\hline Use of steroids anytime during admission, $\mathrm{n}(\%)$ & $17(55)$ & $16(35)$ & 0.081 \\
\hline Chronic kidney disease, $\mathrm{n}(\%)$ & $5(16)$ & $20(43)$ & 0.010 \\
\hline CRRT, n (\%) & $4(13)$ & $25(54)$ & 0.0001 \\
\hline Use of inotropes, $n(\%)$ & $3(9)$ & $26(56)$ & 0.0001 \\
\hline Duration of vasopressors support, days, median & 8 & 19 & 0.0001 \\
\hline Duration of mechanical ventilation, days, median & 23 & 35 & 0.251 \\
\hline $\begin{array}{l}\text { Hospital length of stay prior to infection, days, median, } \\
\text { (IQR) }\end{array}$ & $12(5,36)$ & $34(16,58)$ & 0.018 \\
\hline Hospital length of stay, days, median & $\begin{array}{l}96 \\
(34,139)\end{array}$ & $65(32,112)$ & 0.081 \\
\hline ICU length of stay, days, median, (IQR) & $37(16,67)$ & $46(20,88)$ & 0.465 \\
\hline Admission to index culture, days, median & 10 & 15 & 0.075 \\
\hline Duration of empirical antibiotic therapy, days, median & 12.5 & 7 & 0.031 \\
\hline Duration of definite antibiotics therapy, days, median & 14 & 12 & 0.116 \\
\hline Index organism being MDR first cultured, n (\%) & $19(61)$ & $34(74)$ & 0.243 \\
\hline No. of antibiotics used empirically, median & 2 & 2 & 0.772 \\
\hline Appropriate empirical antibiotics choice, $\mathrm{n}(\%)$ & $24(77)$ & $34(74)$ & 0.923 \\
\hline No. of antibiotics used after sensitivity, median & 2 & 2 & 0.369 \\
\hline
\end{tabular}

Table 5: Multivariate regression analysis for factors associated with mortality. 


\begin{tabular}{|llll|}
\hline Variable & OR & $95 \%$ Cl & P-value \\
\hline Age & 1.07 & $(1.0,1.16)$ & 0.039 \\
\hline APACHE II score $1^{\text {st }} 24$ hours in ICU & 1.13 & $(0.85,1.50)$ & 0.382 \\
\hline APACHE IV score $1^{\text {st }}$ 24 hours in ICU & 1.03 & $(1.0,1.06)$ & 0.04 \\
\hline SOFA score $1^{\text {st }} 24$ hours in ICU & 0.91 & $(0.60,1.36)$ & 0.660 \\
\hline Presence of infection on ICU admission, n (\%) & 0.44 & $(0.02,7.58)$ & 0.573 \\
\hline Chronic kidney disease & 2.43 & $(0.13,44.07)$ & 0.548 \\
\hline Use of inotropes & 10.01 & $(1.55,64.77)$ & 0.015 \\
\hline Duration of mechanical ventilation & 0.95 & $(0.84,1.07)$ & 0.448 \\
\hline ICU length of stay prior to infection & 1.02 & $(0.91,1.15)$ & 0.635 \\
\hline ICU length of stay & 1.03 & $(1.00,1.06)$ & 0.035 \\
\hline Hospital length of stay & 0.97 & $(0.95,0.99)$ & 0.009 \\
\hline Index organism MDR first cultured & 1.01 & $(0.08,11.93)$ & 0.992 \\
\hline Presence of hospital acquired pneumonia & 100.13 & $(2.50,3996.04)$ & 0.412 \\
\hline Presence of CLABSI & 0.35 & $(0.02,4.28)$ & 0.276 \\
\hline
\end{tabular}

\section{Figures}


255 screened for inclusion

\section{Exclusion}

34 cases colonization only

8 cases died on same day being included

136 cases didn't meet MDR definition

\section{7 met inclusion criteria}

44 didn't survive at day 28 of inclusion

33 survived at day 28 of inclusion

\section{Figure 1}

Flowchart of patients' selection.

255 screened for inclusion

\section{Exclusion}

34 cases colonization only

8 cases died on same day being included

136 cases didn't meet MDR definition

77 met inclusion criteria

44 didn't survive at day 28 of inclusion

33 survived at day 28 of inclusion 
Figure 1

Flowchart of patients' selection.

\begin{tabular}{|l|l|}
\hline \multicolumn{1}{|l|}{255 screened for inclusion } \\
Exclusion \\
\begin{tabular}{|l|l|}
34 cases colonization only \\
8 cases died on same day being included \\
136 cases didn't meet MDR definition
\end{tabular} \\
\hline 44 didn't survive at day 28 of inclusion \\
\hline
\end{tabular}

\section{Figure 1}

Flowchart of patients' selection. 


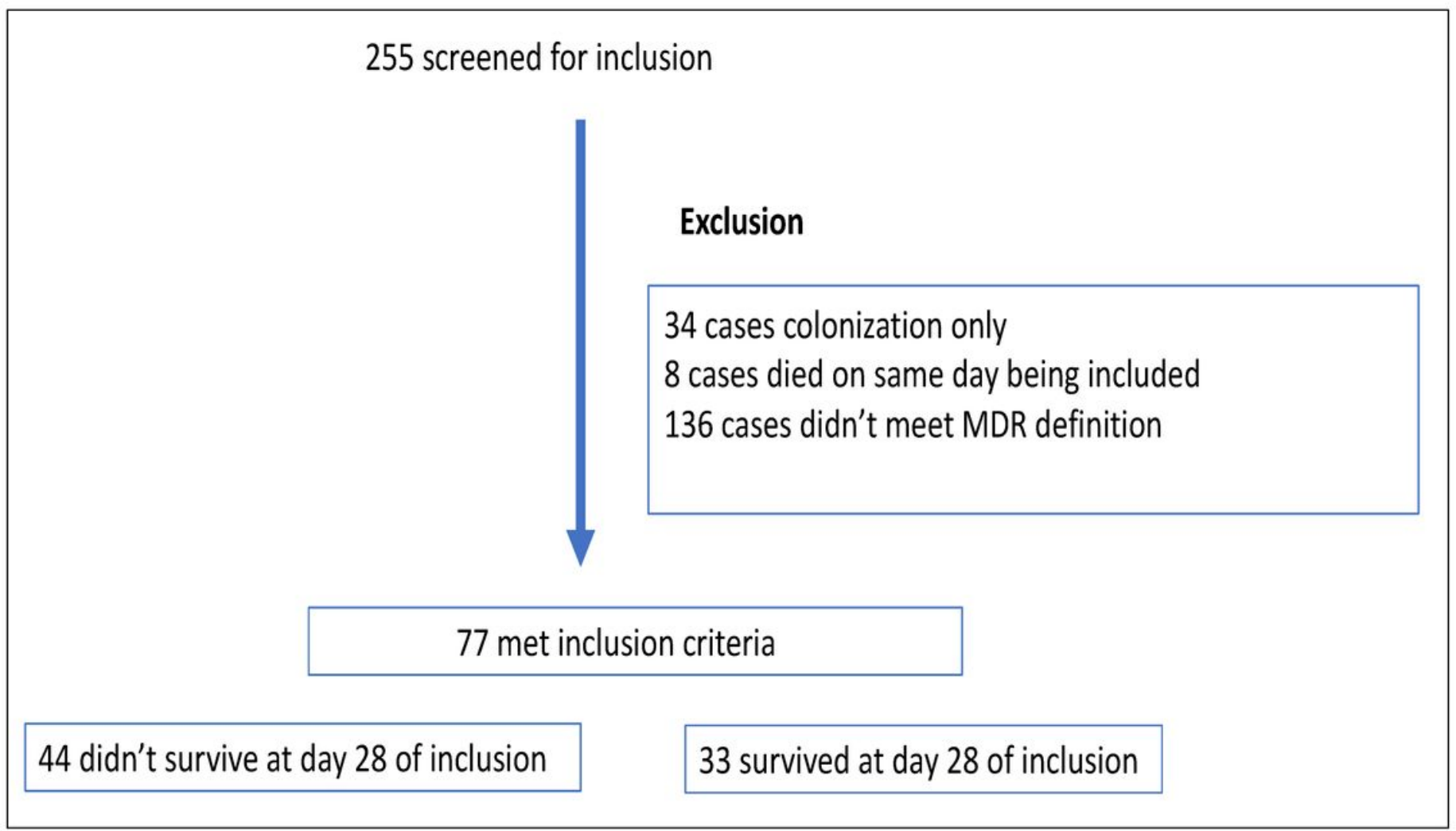

Figure 1

Flowchart of patients' selection. 